\title{
Advances in gringo series 3
}

\author{
Martin Gebser, Roland Kaminski, Arne König, and Torsten Schaub* \\ Institut für Informatik, Universität Potsdam
}

\begin{abstract}
We describe the major new features emerging from a significant redesign of the grounder gringo, building upon a grounding algorithm based on semi-naive database evaluation. Unlike previous versions, rules only need to be safe rather than domain-restricted.
\end{abstract}

\section{Introduction}

A distinguishing feature of Answer Set Programming (ASP; [1]) is its highly declarative modeling language along with its domain-independent grounding systems, like lparse [2], dlv [3], and gringo [4]. This paper is dedicated to the features of the new major release of gringo, starting with version 3. Most notably, this series only stipulates rules to be safe (cf. [5]) rather than $\lambda$-restricted [6], as in previous versions of gringo. Hence, programs are no longer subject to any restriction guaranteeing a finite grounding. Rather, this responsibility is left with the user in order to provide her with the greatest flexibility. This general setting is supported by a grounding algorithm based on semi-naive database evaluation (cf. [5]), closely related to that of $d l v$. In what follows, we elaborate upon the new features emerging from this significant redesign. For a thorough introduction of gringo's language, please consult the manual available at [7].

\section{Advances in gringo series 3}

The most significant change from gringo 2 to 3 is that the basic grounding procedure of gringo 3 no longer instantiates the rules of a logic program strictly along a predefined order. This enables more convenient predicate definitions in terms of (positive) recursion. E.g., consider a $\lambda$-restricted "connected graph design" program:

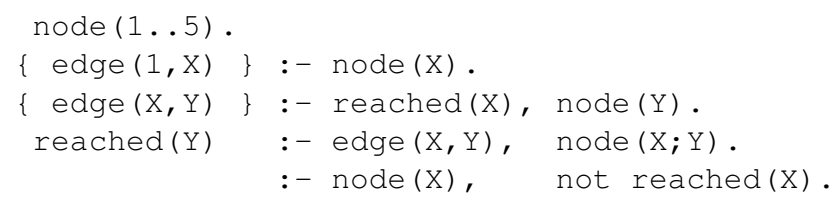

In gringo 3, reached/ 1 can be defined more conveniently as follows:

$\operatorname{reached}(\mathrm{Y}) \quad:-\operatorname{edge}(\mathrm{X}, \mathrm{Y})$.

* Affiliated with Simon Fraser University, Canada, and Griffith University, Australia. 
In fact, additional domain information via node $/ 1$, needed in rule-wise grounding to "break" the cyclic definition of edge/2 and reached/1, is not anymore required in view of semi-naive evaluation.

Since gringo features built-in arithmetics and uninterpreted functions, it deals with potentially infinite Herbrand universes, and the safeness condition does not guarantee termination. For instance, gringo 3 does not terminate on the following program:

$$
\operatorname{succ}(X, X+1):-\operatorname{succ}(X-1, X) \cdot \operatorname{succ}(X, X+1):-\operatorname{zero}(X) \cdot \quad \operatorname{zero}(0) \cdot
$$

In fact, the program has an infinite answer set, and it is not $\lambda$-restricted in view of the first (recursive) rule. Although it may appear disturbing that the termination of gringo 3 is not always guaranteed, we deliberately chose to not reintroduce any syntactic finiteness check, and rather leave it to the user to include appropriate stop conditions limiting the "relevant" ground instances of rules. E.g., one may replace the first rule by:

$$
\operatorname{succ}(X, X+1):-\operatorname{succ}(X-1, X), X<42
$$

Since gringo 3 evaluates built-ins while instantiating a rule, it stops grounding at $\operatorname{succ}(41,42)$, so that only finitely many relevant ground rules are produced.

The design decision to not enforce (syntactic) conditions guaranteeing termination gives users the freedom to write "clever" encodings, where syntactic checks are bound to fail. To see this, consider the following encoding of a universal Turing Machine:

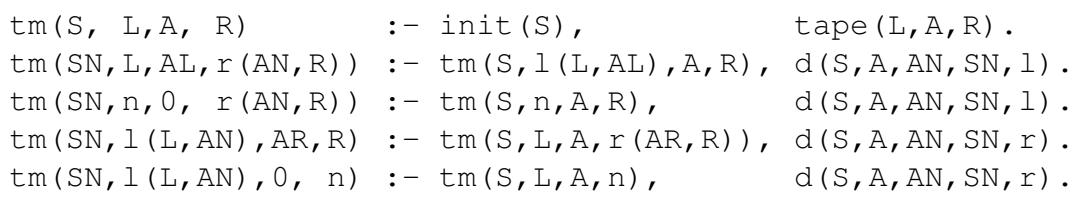

The idea is to represent configurations of the universal Turing Machine by instances of tm (State, LTape, Symbol, RTape), where State is a state of the machine that is run, Symbol is the tape contents at the current read/write-head position, and LTape and RTape are (usually) functions representing the tape contents to the left and right, respectively, of the current position. Starting from an initial state and tape, successor configurations are calculated relative to a transition table given by instances of d (State, Symbol, NSymbol, NState, Direction). For instance, if the direction is 1 for "left," the contents of NSymbol is appended to the tape contents to the right, and the next symbol to the left is taken as the contents at the new position, while also removing it from the left-hand side's tape contents. Hereby, we use n to indicate infinitely many blanks 0 to the left or right of the current position, and dedicated rules take care of "generating" a blank on demand when a tape position is visited first. A machine to run, e.g., a 3-state Busy Beaver machine, can then be given by facts like:

$$
\begin{array}{llll}
d(a, 0,1, b, r) . & d(b, 0,1, a, 1) . & d(c, 0,1, b, 1) . & \operatorname{init}(a) . \\
d(a, 1,1, c, 1) . & d(b, 1,1, b, r) . & d(c, 1,1, h, r) . & \operatorname{tape}(n, 0, n) .
\end{array}
$$

If we run gringo 3 on the universal Turing Machine encoding along with the facts specifying the 3-state Busy Beaver machine, it generates all traversed configurations, where the final one is as follows:

$$
\operatorname{tm}(\mathrm{h}, 1(1(1(1(\mathrm{n}, 1), 1), 1), 1), 1, \mathrm{r}(1, \mathrm{n}))
$$


The fact that gringo 3 terminates tells us that the 3-state Busy Beaver machine halts after writing six times the symbol 1 to the tape. However, given that gringo 3 can be used to simulate any machine and the halting problem, in general, is undecidable, it is also undecidable whether semi-naive evaluation yields a finite grounding, i.e., whether gringo 3 eventually terminates.

The language of gringo 2 [4] already included a number of aggregates, most of which are still supported by gringo 3: \# count, \# sum, \#min, \#max, \#avg, \#even, and \# odd. ${ }^{1}$ The support also includes backward compatibility to the traditional notation of cardinality and weight constraints $[2], 1\{\ldots\} \mathrm{u}$ and $\mathrm{l}[\ldots] \mathrm{u}$, respectively, rather than $1 \#$ count $\{\ldots\} \mathrm{u}$ and $1 \#$ sum [... $]$ u. As with gringo 2 , the condition connective ':' allows for qualifying local variables within an aggregate (or variablesized conjunctions/disjunctions). Hence, gringo 2 and 3 both accept the next program:

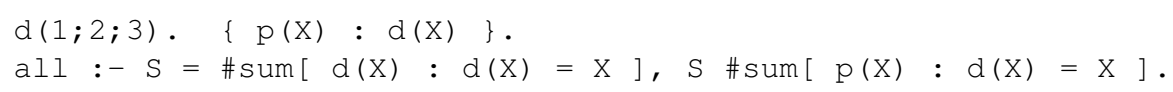

Note that all local variables (named $\mathrm{X}$ ) within aggregates are bound via a domain predicate [2], $d / 1$, on the right-hand side of ':'. While such binding via domain predicates (or built-ins) had been mandatory with gringo 2 , it can often be omitted with gringo 3. E.g., the last rule above can also be written shorter as follows:

all :- $S=\#$ sum $[d(X)=X], S$ \#sum[ $p(X)=X]$.

After investigating the rules with atoms of the predicates $d / 1$ and $p / 1$ in the head and collecting their ground instances, gringo 3 notices that no further rule can derive any instances, so that both domains are limited to 1,2 , and 3. Hence, explicit domain information is not needed to identify all eligible values for the local variables $\mathrm{X}$ in the remaining rule. In fact, since $d / 1$ is a domain predicate, gringo 3 (deterministically) calculates $\mathrm{S}=6$, which is then taken as the lower bound in 6 \# $\mathrm{sum}[\mathrm{p}(1), \mathrm{p}(2), \mathrm{p}(3)]$. However, note that a similar omission of ' $\mathrm{d}(\mathrm{X})$ ' is not admissible in the head of the second rule above, since it would violate the safeness requirement. Finally, gringo 3 does currently not support implicit domains of local variables if an aggregate is involved in (positive) recursion; e.g., the following modified rule is safe, but not yet supported:

$$
\mathrm{p}(\mathrm{S}):-\mathrm{S}=\text { \#sum[ } \mathrm{d}(\mathrm{X})=\mathrm{X}], \mathrm{S} \# \operatorname{sum}[\mathrm{p}(\mathrm{X})=\mathrm{X}] \text {. }
$$

Implicit domains in recursive aggregates are subject to future work (cf. Section 3).

Optimization statements, which can be specified via the directives \#minimize and \#maximize, are syntactically very similar to aggregates, and gringo 3 fully supports implicit domains for local variables in them. (Since optimization statements are not part of logic program rules, they cannot be "applied" to derive any atom.) With lparse and gringo 2, it is possible to provide multiple optimization statements with implicit priorities depending on their order in the input: by convention [2], the last statement is more significant than the second last one, which in turn is more significant then the one before, etc. This convention is also adopted by gringo 3, which must be taken into account when writing a sequence of optimization statements like the following one:

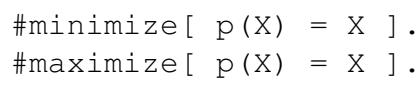
1 The \#times aggregate is currently not supported by gringo 3; while it requires an involved
compilation to "Smodels Internal Format" [2], we are not aware of any application using it. 
According to the order, the \#maximize objective takes precedence over \#minimize. Since such implicit prioritization necessitates a lot of care to be used properly and also undermines the idea of declarative programming, gringo 3 supports explicit priorities via precedence levels, provided via the connective '¿'. This can be used to override default prioritization, e.g., as follows:

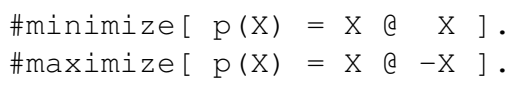

If we assume the domain of $\mathrm{p} / 1$ to contain the values 1,2 , and 3, the corresponding ground optimization statements include the following weighted literals (sorted by their precedence levels):

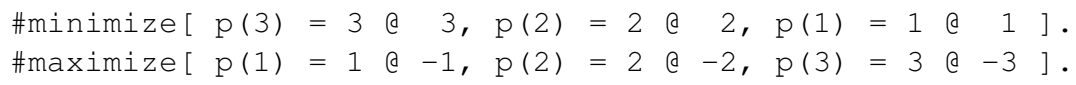

These optimization statements involve six precedence levels, and a greater level is more significant than a smaller one. Accordingly, our main objective is $p(3)$ to be

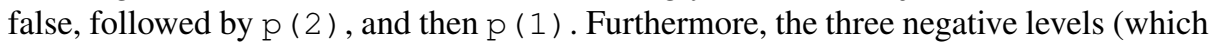
are superseded by the positive ones that are greater) express that we would also like $\mathrm{p}(1)$ to be true, then $\mathrm{p}(2)$, and finally $\mathrm{p}(3)$. Observe that the optimization priorities are fully determined by precedence levels (and weights), so that there are no implicit priorities based on ordering anymore. We note that prioritization of optimization objectives via precedence levels and weights is also supported by $d l v$, which offers weak constraints [3]. In fact, extending gringo by weak constraints is subject to future work.

For selectively displaying atoms, lparse and gringo 2 support \# hide and \# show statements to, at the predicate level, decide which atoms in an answer set ought to be presented or suppressed, respectively. Albeit such output restriction mainly serves user convenience, there are also profound application scenarios, such as the enumeration of projected answer sets [8] offered by clasp (option --project). In fact, the following methodology had been used to, at the predicate level, project Hamiltonian cycles in a clumpy graph down to edges in an underlying "master graph":

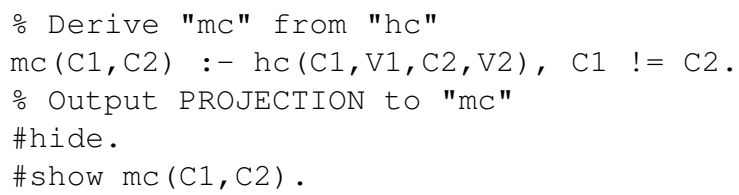

To support output projection not only at the predicate but also at the atom level, gringo 3 allows for conditions, connective ':' followed by domain predicates and/or built-ins, within \#hide and \# show statements. Given this, defining a predicate mc/2 can be omitted and output projection be accomplished more conveniently as follows:

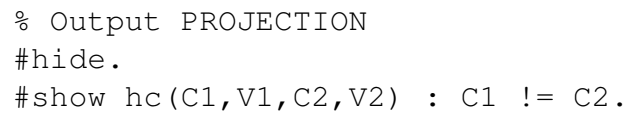

As with precedence levels for optimization, the possibility to distinguish outputs qualified via \#hide and \# show at the atom level, rather than at the level of predicates, contributes to declarativeness, as it abolishes the need to define auxiliary predicates within a logic program only for the sake of projecting the displayed output to them. 
A number of built-in (arithmetic) comparison predicates, viz., '==', '! =', '<=', ' $>=$ ', '<', '>', and (variable) assignments, via '=', were already included in the input language of gringo 2. In gringo 3, respective comparison predicates are generalized to term comparisons, that is, they do not anymore raise an error like "comparing different types," as encountered with (some versions of) gringo 2 when writing, e.g., $2<f$ (a) or alike. Furthermore, while the left-hand side of ' $=$ ' must be a variable, the generalized assignment operator ' $:=$ ' offered by gringo 3 admits composite terms (including variables) on its left-hand side. Hence, it is possible to simultaneously assign multiple variables in a rule like the following one:

$\mathrm{p}(\mathrm{X}, \mathrm{Y}, \mathrm{Z}):-(X, \mathrm{f}(\mathrm{Y}, \mathrm{a}, \mathrm{Z})):=(\mathrm{a}, \mathrm{f}(\mathrm{b}, \mathrm{a}, \mathrm{c}))$.

As with '=', we still require a right-hand side of ' $:=$ ' to be instantiable before ' $:=$, is evaluated. E.g., a rule like the following one is currently not supported by gringo:

$\mathrm{p}(\mathrm{X}):-(\mathrm{X}, \mathrm{a}):=(\mathrm{a}, \mathrm{X})$.

Sometimes, the built-ins offered by a grounder may be too spartan to accomplish sophisticated calculations, and encoding them may likewise be involved and possibly too space-consuming. To nonetheless allow users to accomplish application-specific calculations during grounding, gringo 3 comes along with an embedded scripting language, viz., lua [9]. For instance, the greatest common divisor of numbers given by instances of a predicate $\mathrm{p} / 1$ can be calculated via lua and then be "saved" in the third argument of a predicate $q / 3$, as done in the following program:

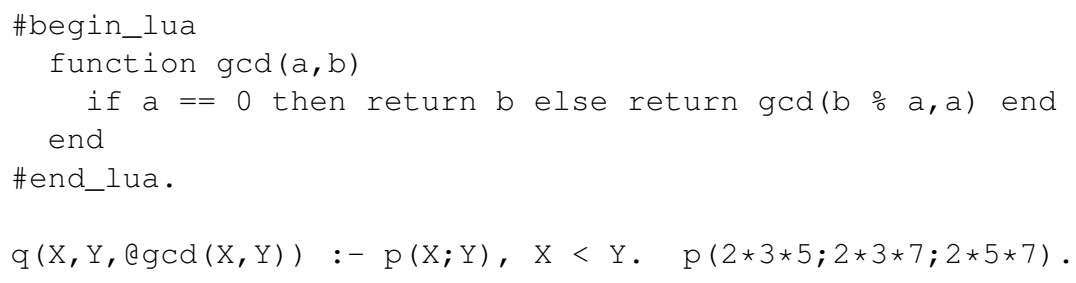

When passing this program to gringo 3 , it for one calculates the numbers being arguments of predicate $\mathrm{p} / 1,30,42$, and 70 , while the implementation of the gcd function in lua is used to derive the following facts over predicate $q / 3$ :

$$
q(30,42,6) . \quad q(30,70,10) . \quad q(42,70,14) .
$$

Beyond sophisticated arithmetics, lua also allows for environment interaction. E.g., it provides interfaces to read off values from a database. In the following example, we use sqlite3, embedded into the precompiled gringo 3 binaries available at [7]:

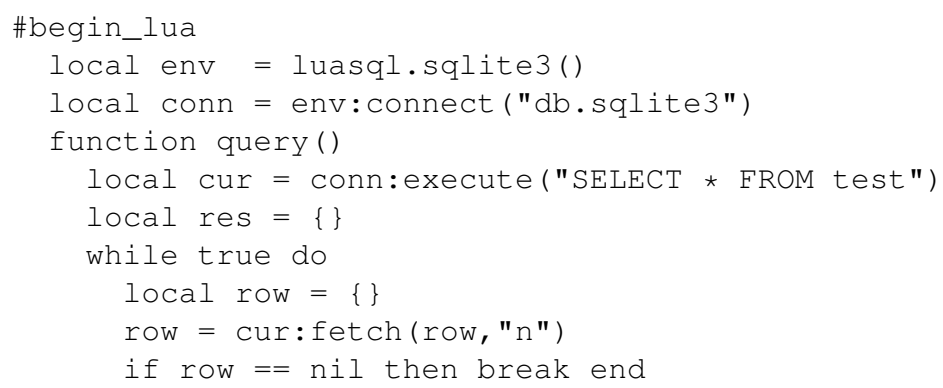




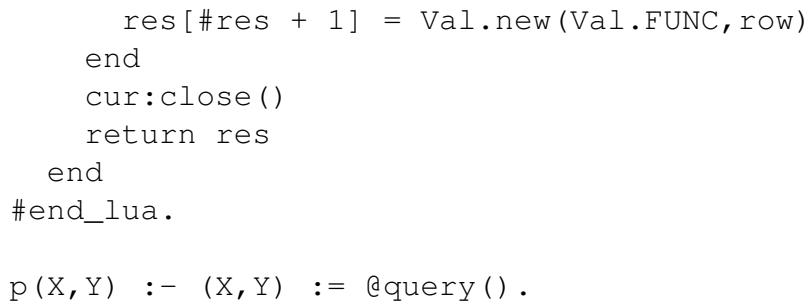

Here, a lua function query is used to read data from a table called test. Although we do here not delve into the details of lua, there is one line that deserves attention:

res[\#res + 1] = Val.new (Val.FUNC, row)

If test contains the tuples $\langle 1, a\rangle,\langle 2, b\rangle$, and $\langle 3, c\rangle$, they are successively inserted into the array res. The collected tuples are then taken to construct the following facts:

$\mathrm{p}(" 1 ", " \mathrm{a} ")$. p ("2","b") . p ("3","c") .

We note that the generation of terms via lua is similar to "value invention" [10], allowing for custom built-in predicates evaluated during grounding.

\section{Discussion}

The redesign of gringo is an important step in consolidating the distinct grounding approaches originated by lparse and $d l v$. A common ASP language unifying the constructs of both approaches is already envisaged as a joint effort of the teams at Calabria and Potsdam. Although $d l v$ and gringo now share many commonalities, like safety, semi-naive database evaluation, function symbols, and Turing completeness, they still differ in aspects like finiteness criteria, indexing, connectivity, incrementality, recursive aggregates, backtracking and -jumping. Hence, it is interesting future work to further investigate the different designs and consolidate them wherever possible.

Acknowledgments. This work was partly funded by DFG grant SCHA 550/8-2.

\section{References}

1. Baral, C.: Knowledge Representation, Reasoning and Declarative Problem Solving. Cambridge University Press (2003)

2. Syrjänen, T.: Lparse 1.0 user's manual. http://www.tcs.hut.fi/Software/smodels/lparse.ps.gz

3. Leone, N., Pfeifer, G., Faber, W., Eiter, T., Gottlob, G., Perri, S., Scarcello, F.: The DLV system for knowledge representation and reasoning. ACM TOCL 7(3) (2006) 499-562

4. Gebser, M., Kaminski, R., Ostrowski, M., Schaub, T., Thiele, S.: On the input language of ASP grounder gringo. In LPNMR'09. Springer (2009) 502-508

5. Abiteboul, S., Hull, R., Vianu, V.: Foundations of Databases. Addison-Wesley (1995)

6. Gebser, M., Schaub, T., Thiele, S.: Gringo: A new grounder for answer set programming. In LPNMR'07. Springer (2007) 266-271

7. http://potassco.sourceforge. net

8. Gebser, M., Kaufmann, B., Schaub, T.: Solution enumeration for projected Boolean search problems. In CPAIOR'09. Springer (2009) 71-86

9. Ierusalimschy, R.: Programming in Lua. http://www.lua.org (2006)

10. Calimeri, F., Cozza, S., Ianni, G.: External sources of knowledge and value invention in logic programming. AMAI 50(3-4) (2007) 333-361 